\title{
Bronchobiliary fistula after multiple transcatheter arterial chemoembolizations for hepatocellular carcinoma: A case report
}

\author{
CAOYE WANG $^{1}$, ZHENGQIANG YANG $^{2}$, JINGUO XIA $^{2}$, WEIPING WANG $^{3}$, WENHUA CHEN $^{1}$ and QI WANG ${ }^{1}$ \\ ${ }^{1}$ Department of Interventional Radiology, First People's Hospital of Changzhou, Changzhou, Jiangsu 213000; \\ ${ }^{2}$ Department of Interventional Radiology, First Affiliated Hospital of Nanjing Medical University, Nanjing, \\ Jiangsu 210029, P.R. China; ${ }^{3}$ Department of Radiology, Mayo Clinic, Jacksonville, FL 32224, USA
}

Received October 31, 2017; Accepted February 5, 2018

DOI: $10.3892 / \mathrm{mco} .2018 .1574$

\begin{abstract}
Bronchobiliary fistula (BBF) is a rare condition, defined as an abnormal communication between the bronchial system and the biliary tree. Patients with this condition usually present with massive biliptysis, and the mortality rate is high. $\mathrm{BBF}$ has been reported to occur in patients with congenital conditions, complications of trauma, hepatic abscesses and biliary tract obstruction (surgical as well as non-surgical). However, to the best of our knowledge, BBF as a complication of transcatheter arterial chemoembolization (TACE) for hepatocellular carcinoma (HCC) has not been reported to date. We herein report a case of BBF developing as a complication following TACE in a 71-year-old male patient with HCC. The patient was treated by placement of a metallic biliary stent followed by percutaneous transhepatic biliary drainage to decompress the intrahepatic biliary tree, and his symptoms were immediately relieved.
\end{abstract}

\section{Introduction}

Transarterial chemoembolization (TACE) is a well-established intervention for the treatment of hepatocellular carcinoma (HCC). However, TACE has also been associated with a variety of complications, such as right upper quadrant pain, fever, elevated liver enzyme levels and hepatic duct ischemia (1). Bronchobiliary fistula (BBF) is commonly associated with congenital conditions, trauma complications, hepatic abscesses and biliary tract obstruction, and it is a potentially serious condition, associated with a high mortality and morbidity rate (12.2\%) (2). We herein present the case of a patient who developed $\mathrm{BBF}$ as a complication following multiple TACEs

Correspondence to: Dr Zhengqiang Yang, Department of Interventional Radiology, First Affiliated Hospital of Nanjing Medical University, Nanjing, Jiangsu 210029, P.R. China

E-mail: yangzhengqiang@jsph.org.cn

Key words: bronchobiliary fistula, transcatheter arterial chemoembolization, biliary drainage for HCC. To the best of our knowledge, this is the first such case reported in the literature to date.

\section{Case report}

A 71-year-old man with hepatocellular carcinoma (HCC) presented with a 2-day history of persistent cough and sudden-onset expectoration of a large amount of greenish sputum. We was admitted to Li Yang People's Hospital on the 19th of March 2016. The patient reported that the sputum tasted bitter, similar to regurgitated stomach contents. The patient had undergone left lobectomy for HCC 11 years prior, and 19 subsequent TACE procedures. However, the tumor had progressed and compressed the intrahepatic bile duct. A computed tomography (CT) scan of the abdomen performed at a different institute revealed intrahepatic bile duct dilatation; endobronchial presence of a high-density contrast agent in agreement with the density of the intrahepatic bile ducts further confirmed the presence of a bronchobiliary shunt. Other findings were unremarkable. The patient was referred to the Department of Interventional Radiology, First Affiliated Hospital of Nanjing Medical University (Nanjing, China) for further evaluation. Percutaneous transhepatic cholangiography (PTC) was performed, based on the patient's clinical symptoms and intrahepatic duct dilation on CT, and revealed focal high-grade stenosis at the hepatic hilum associated with a severely dilated biliary tree. An incidental finding of faint contrast material at the lower lobe of the right lung was also observed during cholangiography (Fig. 1); this contrast material was suspected to represent contrast extravasation from the biliary system to the lung. A follow-up chest CT revealed contrast material within the bronchial tree of the right lower lung, a finding consistent with the presence of a bronchobiliary shunt (Fig. 2).

A decision was made to establish percutaneous transhepatic biliary drainage to decompress the intrahepatic biliary tree. An 8.5-Fr internal-external biliary drainage tube was placed, and the patient's cough was relieved on the following day. The patient was admitted for routine catheter replacement 2 months later, and cholangiography revealed interval resolution of the contrast extravasation from the biliary system to the lung. As a palliative measure, the biliary drain was removed and a metallic biliary stent (E-Luminex; Bard, Murray Hill, 
NJ, USA) was placed to relieve the hilar stenosis (Fig. 3). The patient remained asymptomatic after the stent placement until he eventually succumbed to liver failure, infection, and underlying tumor progression 3 months later.

\section{Discussion}

BBF is the formation of an abnormal communication between the biliary system and the bronchial tree. The mechanism of $\mathrm{BBF}$ remains speculative due to the rarity of this condition. The most commonly accepted hypothesis is an elevation of the biliary system pressure, which produces an inflammatory reaction in the subdiaphragmatic space and subsequent rupture into the bronchial tree $(3,4)$. Locally aggressive processes, such as tumor infiltration or abscess rupture through the diaphragm, are other possible causes of BBF (5). Warren et al (6) suggested that two conditions must be met before a BBF occurs in patients with biliary tract disease: Partial or complete obstruction of the bile duct, and formation of adhesions between the lung and the diaphragm. In the present case, the patient had a hilar obstruction and had undergone multiple TACE treatments, particularly in the dome area, which may have caused tissue necrosis. Once the intrabiliary pressure increases, bile may leak from the necrotic area, rupture through the liver capsule and diaphragm, and eventually erode into the lung and bronchial tree.

The diagnosis of BBF is straightforward, but a high level of clinical suspicion is required. The symptoms include an irritating cough, yellowish sputum and symptoms of acute bronchitis (7). Sputum analysis should show a bile component, rather than the typical inflammatory process characterized by large numbers of bacteria and white cells. PTC and endoscopic retrograde cholangiopancreatography (ERCP) remain the procedures of choice to determine the exact anatomy of the fistula (8). Radiological imaging (thoracic and upper abdominal CT) may be required to confirm the presence of contrast material within the bronchial tree after T-tube cholangiography. Identification of the underlying fistula is useful to optimize the treatment strategy, which involves either resection of the fistula or embolization with minimally invasive techniques. In the present case, the fistula was located in the dome of the liver and the drainage tube was placed through the right intrahepatic system, which appeared to adequately decompress the bile from the hilar obstruction.

The management of BBF may be surgical or non-surgical (PTC or ERCP), and a well-planned management strategy, including proper antibiotics and supportive care, is required. Surgical approach is a definitive treatment, but requires thoracotomy and/or laparotomy with resection of the fistula and re-establishment of normal biliary drainage, and such procedures are often associated with high morbidity (14.3\%) and mortality rates (12.7\%) (9). Non-surgical management includes percutaneous or endoscopic placement of a biliary drain and/or stent to decompress the intrahepatic biliary tree by diverting the bile. Such techniques are less invasive and can provide good results with lower morbidity. Our patient was not a surgical candidate due to his extensive disease and overall poor medical condition; therefore, placement of a biliary drain was considered the treatment of choice. The drain appeared to effectively decompress the biliary tract pressure with

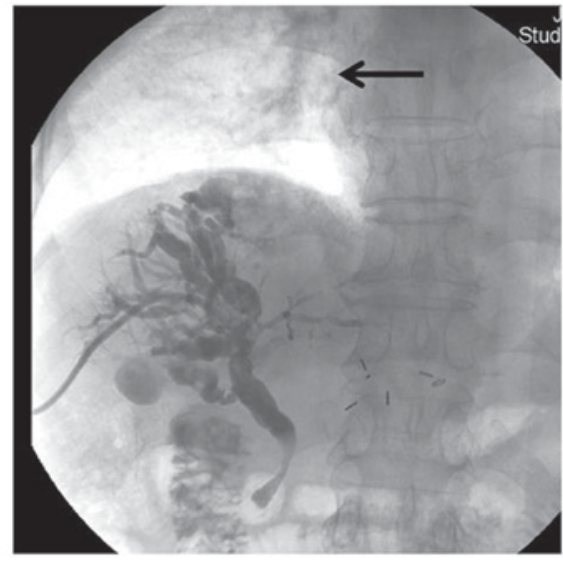

Figure 1. Percutaneous transhepatic cholangiography revealed right-sided bronchial enhancement (arrow) followed by intrahepatic biliary tract dilation and right-sided bile duct expansion.

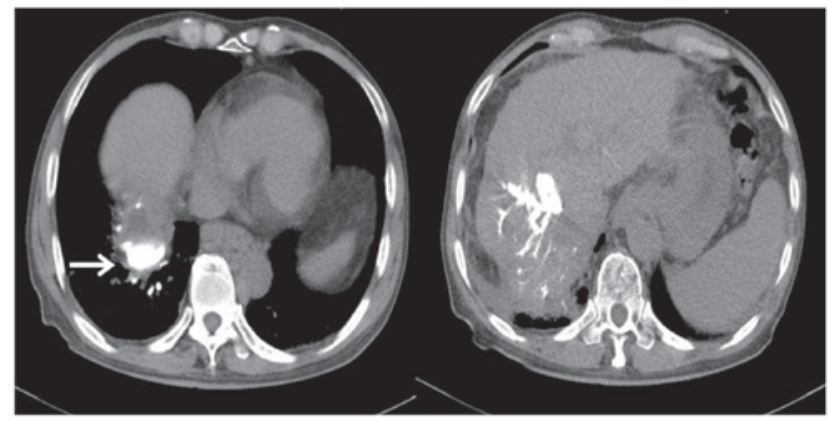

Figure 2. Computed tomography (CT) was performed immediately after percutaneous transhepatic cholangiography. Plain axial CT revealed the presence of endobronchial contrast media (left panel, arrow), in agreement with the density of the intrahepatic bile ducts (right panel), which further confirmed the diagnosis of bronchobiliary fistula.

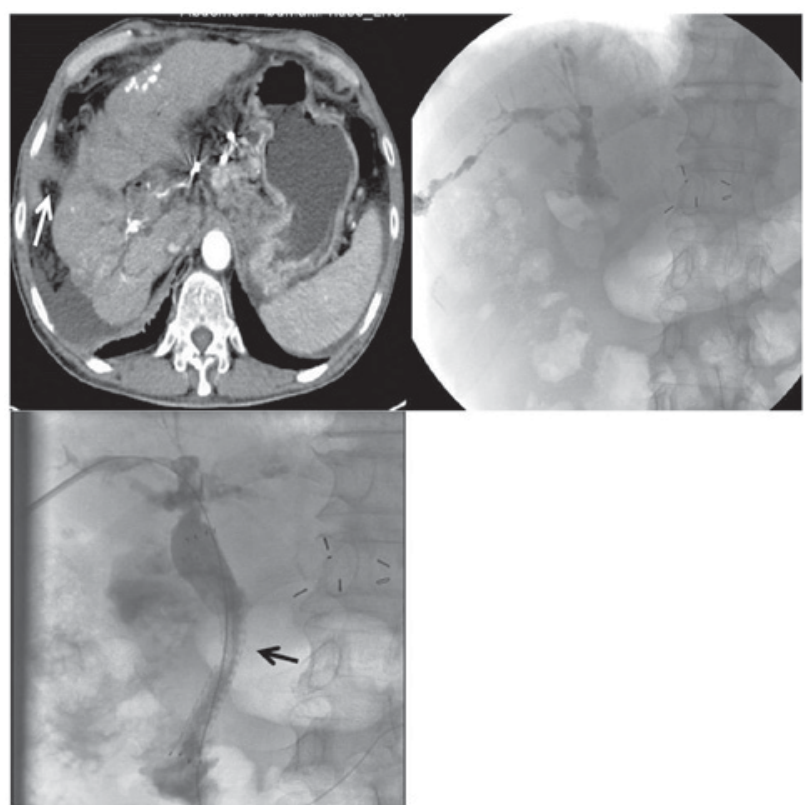

Figure 3. A computed tomography scan revealed bile duct obstruction and biliary sinus formation (white arrow, top left image). A metallic biliary stent (black arrow) was placed across the stricture, which was located in the hepatic hilum (bottom left image). A computed tomography scan revealed bile duct obstruction in the hepatic hilum after injecting the contrast media (top right image). 
immediate cessation of the biliptysis. However, we did not further attempt to close the fistula tract using embolic material or any devices, as the patient's clinical symptoms had resolved following drain placement. Thus, decompression of the biliary tract may be the key to management of BBF in the emergency setting. In the present case, the patient had undergone multiple (19 times) subsequent TACE procedures. Such cases are at a high risk of BBF, as after several TACEs the blood supply of the bile duct may become compromised, with ensuing high pressure, ischemia and necrosis, leading to BBF formation.

In conclusion, $\mathrm{BBF}$ is an uncommon condition that is associated with severe mortality and morbidity. Imaging confirmation of an abnormal communication between the biliary and bronchial trees is required to establish the diagnosis, and PTC or ERCP are the most common treatment choices. A CT scan may reveal the presence of contrast material in the bronchial tree following cholangiography. The management of BBF may be surgical or non-surgical, aimed at either permanent resection of the fistula or palliative decompression of the biliary tree, depending on each individual case.

\section{Acknowledgements}

The authors wish to thank Angela Morben, DVM, ELS, from Liwen Bianji, Edanz Group China, for editing the English text of a draft of this manuscript.

\section{Funding}

The present study received funding from the Yong Research Project of First People's Hospital of Changzhou.

\section{Availability of data and materials}

Not applicable.

\section{Authors' contributions}

CW, ZY made substantial contributions to conception and design; WC and QW did acquisition of data, analysis and interpretation of data; CW, JX, WW were involved in drafting the manuscript and revising it critically for important intellectual content; $\mathrm{CW}$ and $\mathrm{ZY}$ gave final approval of the version to be published. Each author has participated sufficiently in the work to take public responsibility for appropriate portions of the content; and agreed to be accountable for all aspects of the work in ensuring that questions related to the accuracy and integrity of any part of the work are appropriately investigated and resolved.

\section{Ethics approval}

This article does not contain any studies with human participants or animals performed by any of the authors.

\section{Consent for publication}

The patient and/or his family consented to the publication of the case details and associated images.

\section{Competing interests}

The authors declare that they have no competing interests.

\section{References}

1. Miyayama S and Matsui O: Superselective conventional transarterial chemoembolization for hepatocellular carcinoma: Rationale, technique and outcome. J Vasc Interv Radiol 27: 1269-1278, 2016.

2. Moumen M and el Fares F: Bilio-bronchial fistula of hydatid origin. Apropos of 8 cases. J Chir (Paris) 128: 188-192, 1991 (In French).

3. Singh B, Moodley J, Sheik-Gafoor MH, Dhooma N and Reddi A: Conservative management of thoracobiliary fistula. Ann Thorac Surg 73: 1088-1091, 2002.

4. Ferguson TB and Burford TH: Pleurobiliary and bronchobiliary fistulas. Surgical management. Arch Surg 95: 380-386, 1967.

5. Kim YS, Rhim H, Sung JH, Kim SK, Kim Y, Koh BH, Cho OK and Kwon SJ: Bronchobiliary fistula after radiofrequency thermal ablation of hepatic tumor. J Vasc Interv Radiol 16: 407-410, 2005.

6. Warren KW, Christophi C, Armendariz R and Basu S: Surgical treatment of bronchobiliary fistulas. Surg Gynecol Obstet 157: 351-356, 1983.

7. Gugenheim J, Ciardullo $\mathrm{M}$, Traynor $\mathrm{O}$ and Bismuth $\mathrm{H}$ : Bronchobiliary fistulas in adults. Ann Surg 207: 90-94, 1988.

8. Rose DM, Rose AT, Chapman WC, Wright JK, Lopez RR and Pinson CW: Management of bronchobiliary fistula as a late complication of hepatic resection. Am Surg 64: 873-876, 1998.

9. Kabiri H, Chafik A, al Aziz S, el Maslout A and Benosman A: Treatment of hydatid bilio-bronchial and bilio-pleuro-bronchial fistulas by thoracotomy. Ann Chir 125: 654-659, 2000 (In French). 